\title{
Whirling System of Water Exchange in Breeding Pools
}

\author{
Karolina Matej ${ }^{1}$, Iwona Pawliczka ${ }^{2}$, Jerzy M. Sawicki ${ }^{1}$, \\ Pawel Wielgat ${ }^{1}$, Piotr Zima*1
}

\author{
${ }^{1}$ Gdańsk University of Technology, Faculty of Civil and Environmental Engineering, ul. G. Narutowicza \\ 11/12, 80-233 Gdańsk, Poland \\ ${ }^{2}$ University of Gdańsk, Institute of Oceanology, Marine Station, ul. Morska 2, 84-159 Hel, Poland \\ ${ }^{*}$ Corresponding author \\ E-mails: karolina.matej@gmail.com, iwona.pvp@ug.edu.pl,jsaw@pg.gda.pl,pawwielg@pg.gda.pl, \\ pzim@pg.gda.pl
}

(Received December 12, 2016; revised March 16, 2017)

\begin{abstract}
To create proper living conditions for sea mammals kept in closed systems, one has to make sure that the characteristics of breeding pools, such as their shape, dimensions, the facing of the walls and bottom, as well as the quality and motion of water, resemble as closely as possible the natural environment of the animals. An appropriate system of water exchange plays a very important role here. A complete exchange of water is time-consuming, expensive and troublesome, so it can be performed only periodically and should be supported by a supplementary continuous exchange. This operation improves water quality and can create a proper velocity field in breeding pools. The breeding pools investigated in the present study are located in a sealarium in Hel (Poland), which belongs to the Institute of Oceanography of the Gdańsk University. Tracer measurements, carried out in these reservoirs made it possible to evaluate the intensity of continuous water exchange. It was found that this intensity was insufficient (as evidenced by large dead zones in the pools and short detention time), and therefore alterations to the existing system were proposed (i.e. a tangential position of the inlet and a centrally situated outlet). On the basis of a simplified model of circulative water flow, it was shown that the altered hydraulic system can considerably improve the situation.
\end{abstract}

Key words: sea mammal breeding; sealarium; water circulation, breeding pools

\section{List of symbols}

$B \quad-$ semi-empirical multiplier $\left[\mathrm{m}^{1.65} / \mathrm{s}\right]$;

$c_{A} \quad-$ average concentration of the tracer $\left[\mathrm{mg} / \mathrm{m}^{3}\right]$;

$c_{M}-$ mean concentration of the tracer (plug flow time) $\left[\mathrm{mg} / \mathrm{m}^{3}\right]$;

$c_{T} \quad-$ terminal concentration of the tracer in the outlet $\left[\mathrm{mg} / \mathrm{m}^{3}\right]$;

$\operatorname{dim}$ - dimension $[-]$;

$E(t)-$ distribution of the standard retention time [-]; 


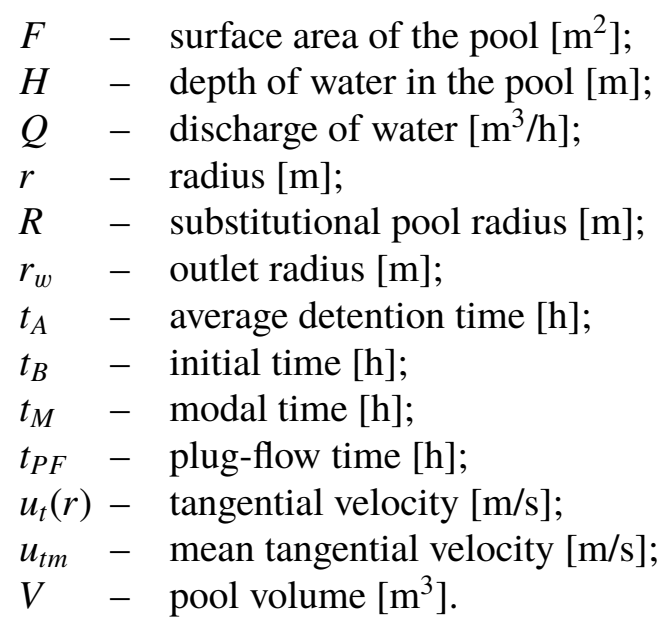

\section{Introduction}

The deterioration of natural environment caused by human activity creates many problems and difficulties. Different ecological factors superimpose, which strengthens their negative effects. In consequence, the existence of some species of animals and plants is endangered. Hence, some corrective activities, are necessary.

A particular set of urgent problems affects sea mammals. This specific category of animals, containing more than 120 recognized species, can be subdivided into four groups:

- Cetaceans (whales, dolphins, porpoises);

- Sirenians ( manates, dugongs);

- Pinnipeds (seals, sea lions, walruses);

- Fissipeds (polar bears, otters).

In addition to the above-mentioned environmental factors (such as degradation and pollution of the animals' habitat, including global climate changes), these animals are also endangered by the excessive exploitation of sea resources. Originally, marine mammals were hunted by some aboriginal tribes for meat, fat, fur and bones. Efforts have been made to restrain or reduce hunting, but these animals are still a very attractive commodity and constitute a significant part of the food market in some countries.

Yet it is not hunting alone that contributes to the decline in sea mammal populations. By-catch (i.e. incidental capture by nets), collisions with ships, economical conflicts with fishery and aquaculture (for both food and space) also play an important role. The last of these factors has an ambivalent character, as aquaculture is becoming an increasingly important source of food for people and animals. Therefore, the producers are fully entitled to pursue their interests. The conflict with fishers is not as violent as it used to be (e. g. in the first half of the 20th century, seals in many countries 
were treated as vermin and exterminated), but still in special circumstances these animals, when creating danger situations, may be lethally removed (in a controlled and humane way).

All these conditions briefly outlined above show the complexity of the problem. This paper presents selected results of broad efforts aimed at restoring a herd of grey seals along the Polish coast of the Baltic sea. Specifically, the paper deals with the problem of continuous water exchange in reservoirs and breeding pools in which these animals are bred.

\section{Artificial Habitat of Sea Mammals}

All creatures, not only sea mammals, should live in a possibly favorable environment. In the proper attitude it should be their natural environment, but it would be an idealistic approach, as human activity has left irreparable scars on the face of our globe. In practice, we have to settle (at best) for a geographical environment (with a transmuted natural environment) or for an anthropogenic environment (i.e. one created almost completely by humans). It is obvious that we should try to improve this situation.

Investigations presented in this paper concern artificial facilities and installations in which water animals have to live. Depending on the kind of animals in question (algae, seaweeds, oysters, fish, mammals), we are dealing with different types of facilities (e.g. aquaria, sealaria, dolphinaria, oceanaria, aquaparks, sea parks etc.).

One has to bear in mind that plants and animals are kept there for different reasons. Aside from the production of food or other goods (e.g. artificial pearls) in aquafarms, the most socially accepted reason for these facilities is to restore or improve endangered animal populations and to treat ill or weak animals (e.g. medication, quarantine). Probably a similar level of social acceptance is accorded to centers designed for education, scientific research and medical purposes (e.g. therapy and convalescence of ill and disabled people).

More doubts arouse the existence of recreation and entertainment facilities with sea animals. Many NGOs point out that the animals there are forced to perform exaggerated variations of their natural behavior (as it was expressed by respected UE documents).

Even more controversial are programs of exploiting sea animals for military purposes developed in some countries. However, if are to believe the assurances of the navy, these animals are not trained for strictly military purposes, but merely for some technical tasks, such as naval mine detection, protection of harbors and ships against enemy divers and swimmers, or recovery of lost objects.

It is difficult to specify requirements concerning the needs of sea mammals kept in closed systems. What is more, it would be unrealistic to expect that the list of these needs and conditions can be full and precise. Different kinds of animals live in different sea areas in different parts of the world and often travel very long distances. 
Therefore, every source of information about the proper living conditions of animals should be used, beginning with the EU Regulations (especially No. 834/2007 and No. 89/2008, although they concern organic farming) up to professional and scientific literature. One should accept as a rule that even the smallest piece of information should not be disregarded.

This paper contains a proposal for an improvement of the system of continuous water exchange in breeding pools. It is a highly important issue, as the animals themselves are a major source of water pollution (such as food remains and excrements). A complete exchange of water is expensive and time-consuming, and thus can be performed only periodically. For this reason, an additional hydraulic system for continuous water exchange ("flushing" of the pool) becomes more important and should be designed and operated very accurately.

\section{Breeding Pools of Sealarium in Hel}

The facility under consideration belongs to the Marine Station of the Institute of Oceanology at the University of Gdańsk. As already mentioned, its fundamental purpose is reintroduction a herd of grey seals along the Polish coast of the Baltic sea.

The basic part of the sealarium is a system of 7 interconnected reservoirs (Fig. 1): three large pools, which were investigated in this study (Nos. 3, 4 and 7), and four smaller ones, designated mainly for young seals (cubs). These smaller reservoirs were not investigated, because of their small dimensions.

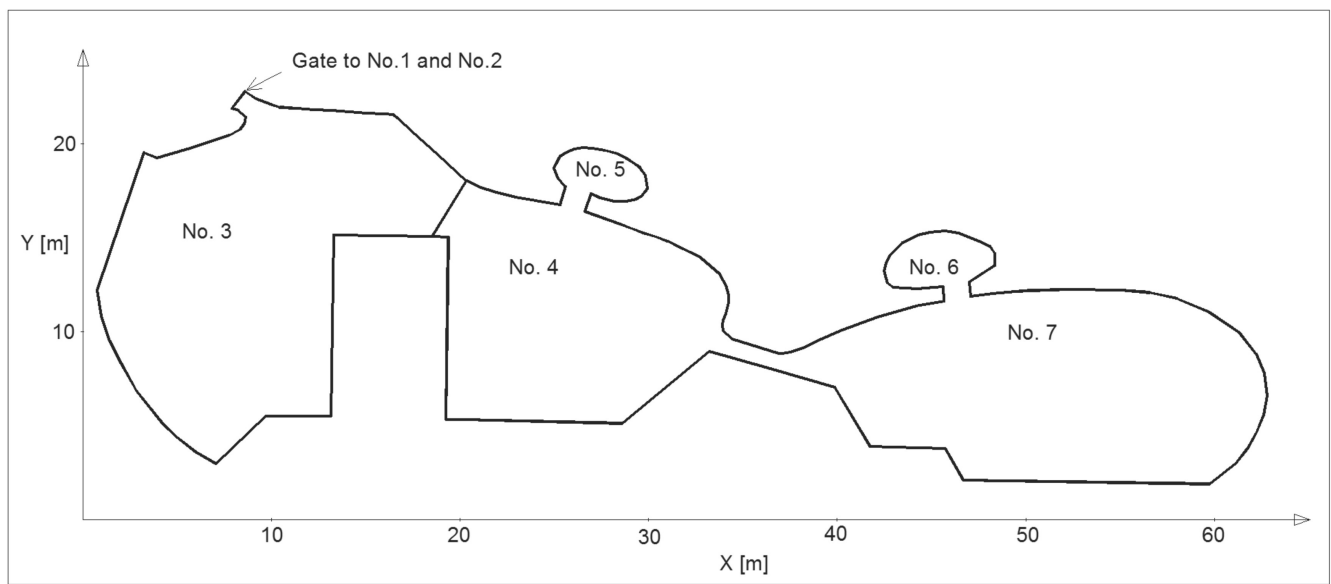

Fig. 1. The layout of breeding pools in the Hel sealarium

The main parameters of these pools are the water depth $H$, the surface area of the pool in a top view $F$, and the discharge of flushing water $Q$. Their values in the pools under investigation are as follows: 


$$
\begin{array}{lll}
H_{3}=2.90 \mathrm{~m} & F_{3}=217 \mathrm{~m}^{2} & Q_{3}=33.80 \mathrm{~m}^{3} / \mathrm{h} \\
H_{4}=1.50 \mathrm{~m} & F_{4}=151 \mathrm{~m}^{2} & Q_{4}=14.00 \mathrm{~m}^{3} / \mathrm{h} \\
H_{7}=1.00 \mathrm{~m} & F_{7}=206 \mathrm{~m}^{2} & Q_{7}=13.25 \mathrm{~m}^{3} / \mathrm{h}
\end{array}
$$

In a simplified, but very popular, approach, a basic parameter that describes the intensity of water motion through a fluid-flow system is the plug-flow time $t_{P F}$. This value is defined as the ratio of the pool volume $V$ to the water discharge $Q$ :

$$
t_{P F}=\frac{V}{Q}=\frac{F H}{Q} .
$$

For the three pools we have:

$$
t_{P F 3}=18.6 \mathrm{~h} \quad t_{P F 4}=16.2 \mathrm{~h} \quad t_{P F 7}=15.5 \mathrm{~h} .
$$

\section{Determination of the Rational Retention Time in Breeding Pools}

The actual "dwelling time" of fluid moving through a flow reactor is described by highly complex mathematical relations, and determination of this parameter is a very difficult task. The plug-flow time (Eq. 4), on the other hand, is very popular in technical applications, but it is a simplified parameter. Therefore, in many situations, it is convenient to use a rational (i.e. as simple formally and as rich in physical meanings as possible) model of this value description. Probably the best of these models is the retention time distribution RTD (Danckwerts 1953).

In the course of research described in this paper, the RTD for the breeding pools was determined empirically by the tracer method (Wilson et al 1986, Sawicki et al 2003). The tracer (Rhodamine WT) was introduced into the inlet of the pool at the initial moment of time $(t=0)$ by the single emptying of a feeder containing $80 \mathrm{ml}$ of the tracer solution (its concentration was $0.2 \mathrm{~g} / \mathrm{dm}^{3}$, so the mass of pure dye in this quantity was $16 \mathrm{mg}$ ). The terminal concentration of the tracer in the outlet $c_{T}(t)$ was measured by a Cyclops 7 (Turner Designs, USA) fluorometer connected to a recording computer.

The measurements were carried out for two pools No. 3 and No. 4 (as the third was occupied by seals). The results obtained are shown in Fig. 2 (pool No. 3) and Fig. 3 (pool No. 4). According to the typical procedure, these functions should be recalculated into the standard retention time distribution curve $E(t)$ by the following relation:

$$
E(t)=\frac{c_{T}(t)}{\int_{0}^{\infty} c_{T}(t) d t} .
$$




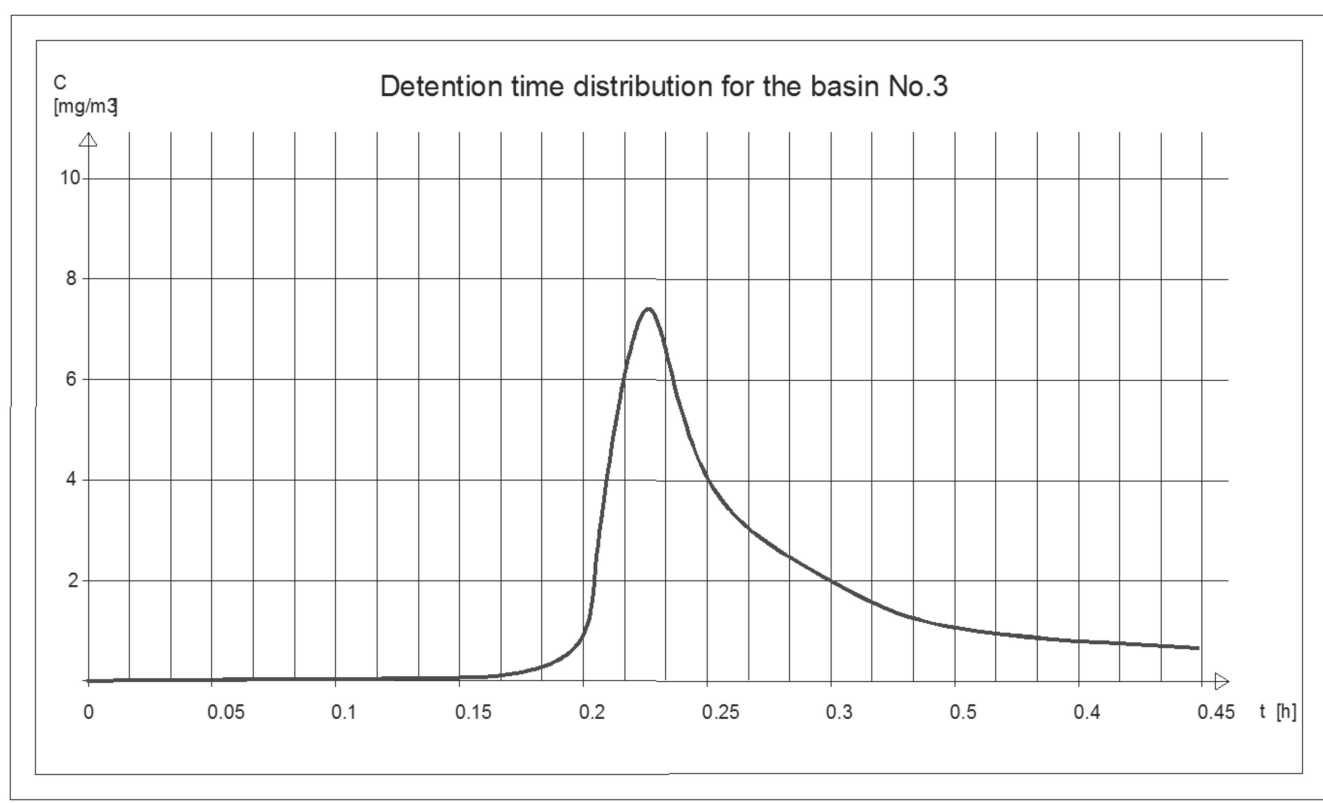

Fig. 2. Retention time distribution for pool No. 3

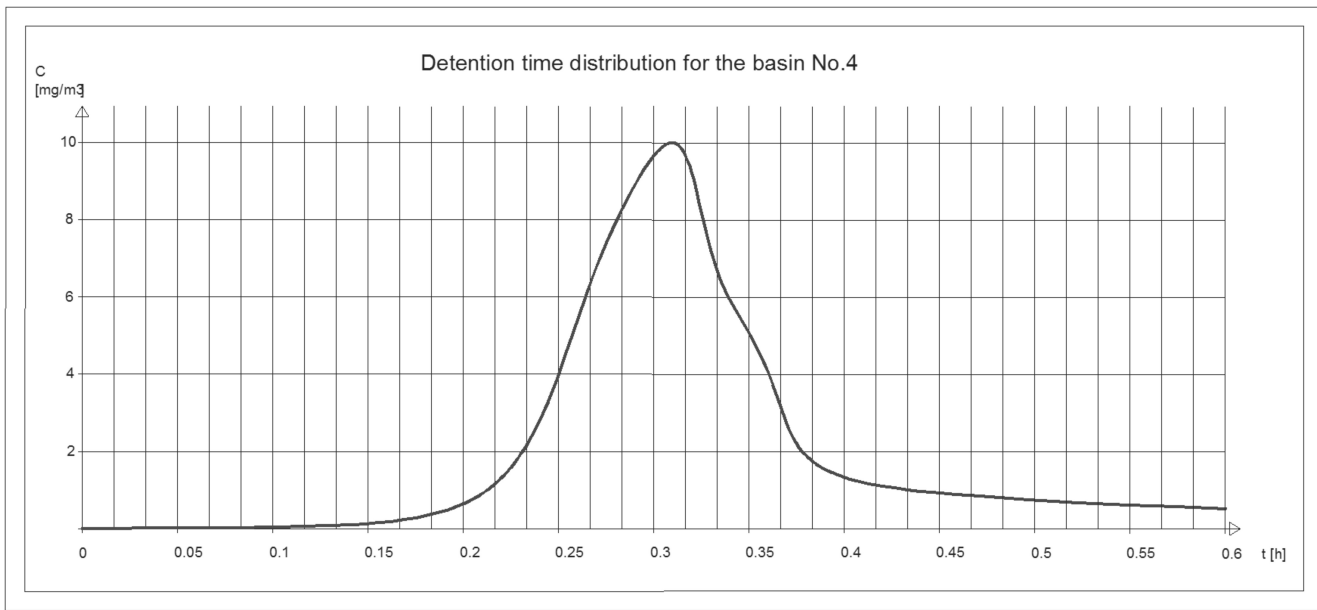

Fig. 3. Retention time distribution for pool No. 4 
Thanks to this normalized function, one can calculate the average retention time $t_{A}$, defined as the first moment of the function $E(t)$ :

$$
t_{A}=\int_{0}^{\infty} t \cdot E(t) d t .
$$

In addition, it is also useful to analyze the initial time $t_{B}$ (when the colored water arrives at the outlet) and the modal time $t_{M}$ (when the maximal concentration of the dye appears at the outlet). In the the present case, the following results were obtained:

$$
\begin{array}{rlrl}
t_{A 3} & =0.42 \mathrm{~h} & t_{B 3}=0.16 \mathrm{~h} & t_{M 3}=0.23 \mathrm{~h}, \\
t_{A 4}=0.56 \mathrm{~h} & t_{B 4}=0.19 \mathrm{~h} & t_{M 43}=0.31 \mathrm{~h} .
\end{array}
$$

Generally, these time characteristics of flow through a reservoir show the actual intensity of water exchange in the system. It can be argued that they describe the true area of the active flow field. The plug-flow time $t_{P F}$, on the other hand, can be interpreted as a simplified parameter, when this active flow field covers the whole pool.

A comparison of these values (Eqs. 8, 9 and Eq. 5) clearly shows that the actual rate of water exchange in these reservoirs is negligible, compared to the level intended by the designers of the sealarium). The average retention time is a minor fraction of the plug-flow time. This means that the flow through the pools has a character of "a hydraulic short circuit". A similar disproportion is seen when comparing the mean concentration of the dye $c_{M}$ that would have been achieved if the tracer had been fully dissolved in water with its actual mean concentration $c_{A}$ in the colored zone of water (Figs. 2, 3). For pool No. 3, $c_{M 3}=0.025 \mathrm{mg} / \mathrm{m}^{3}$, whereas $c_{A 3}$ was close to 3.0 $\mathrm{mg} / \mathrm{m}^{3}$. In the other pool, $c_{M 4}=0.071 \mathrm{mg} / \mathrm{m}^{3}$ and $c_{A 4}$ approached $5.0 \mathrm{mg} / \mathrm{m}^{3}$. Thus, it is evident that the existing system of continuous water exchange does not fulfill its purpose.

\section{Proposed Improvement of the Hydraulic System}

Increasing the flushing water discharge would be an obvious and very simple method to enlarge the active flow zone. However, it would raise the total operating costs of the facility. Moreover, it is not generally known how the animals would respond to locally increased water velocity. For the same reasons, one should exclude the use of local, water-driving devices, such as jet pumps or mixers.

After analyzing the problem, it was concluded that a reasonable solution would be a "whirling" system of continuous water exchange. The essence of this suggested solution is a spiral flow of water from the outer wall of each pool towards the outflow, belonging to the category of "morning glory" overfalls (Nalluri and Featherstone 2001). In order to induce this kind of circulation, the inlet of each pool should be 
oriented tangentially with respect to the outer wall. The outflow, on the other hand, must be situated at the center of the pool bottom (Fig. 4). Obviously, it should be strong enough and shock-proof to withstand contact with seals.

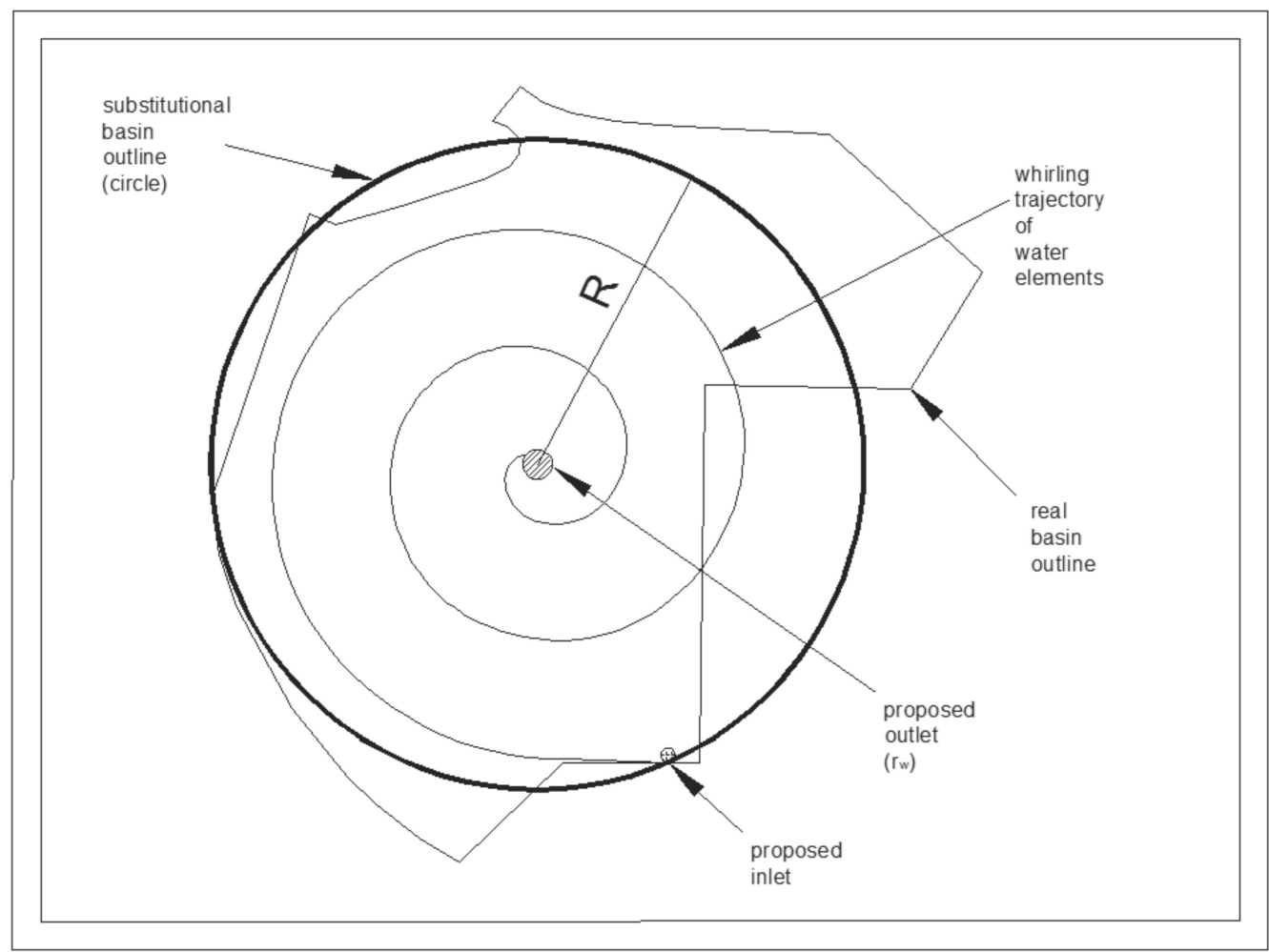

Fig. 4. Schematic diagram of a pool with a whirling flow field

According to the literature (Andoh and Saul 2003), such circulation increases the area of the active flow zone in the reservoir and reduces dead zones. From the quantitative perspective, this effect was described in a dissertation by Gronowska-Szneler (2014). Laboratory measurements in rotational vessels showed that the proportion between the averaged detention time $t_{A}$ and the plug flow time $t_{P F}$ for such facilities is close to 50-60\%, whereas for reservoirs without circulation this ratio hardly reaches $20 \%$. This means that the induction of horizontal rotation increases the area of the active flow zone by 100-200\%.

An accurate mathematical description of flow in the proposed system would be rather difficult and expensive. Keeping this analysis on abasic level, one can use a simplified model of this kind of motion. Originally this model was derived for vortex separators (Gronowska-Szneler and Sawicki 2014), which have regular shapes. Hence, to perform necessary calculations, the pools have to be replaced by cylinders of the 
radius $R$, which have the same plane area $F$ as the original pools (Eqs. 1, 2, 3). The value $R$ can be calculated from the obvious geometrical relation:

$$
R=\sqrt{\frac{F}{\pi}}
$$

For the breeding pools considered here one obtains:

$$
R_{3}=8.3 \mathrm{~m} \quad R_{4}=6.9 \mathrm{~m} \quad R_{7}=8.1 \mathrm{~m} .
$$

The description of the above-mentioned rotational motion belongs to the category of "kinematic" models of fluid flow, intermediate between "differential" and "hydraulic" ones.

The first group (i.e. differential) comprises technical calculation tools, that are placed on a high level of physical accuracy, but are rather complicated mathematically (usually expressed by partial differential equations). The problem formulation (i.e. boundary and initial conditions) in itself is difficult, to say nothing about the solution, which is a time-consuming process, requiring special qualifications from the staff (CFD methods) (Sawicki 2014).

The much more popular hydraulic models, on the other hand, have the form of simple algebraic formulae based on many simplifications and are very often empirical. They provide means of solving many standard technical problems, but are inadequate for more complex flow cases.

In this situation, the kinematic models (Sawicki 1989) become very attractive. The name of this category describes its essential features: the velocity of the flow is described by "kinematic," usually empirical, evaluation of the phenomenon,. The best example of such a model is the potential plane flow (where the absence of visible vortices in the moving fluid leads to the formal condition that $\operatorname{rot} \boldsymbol{u}=0$ ).

The model applied in this paper is based on the following assumptions:

- the flow is two-dimensional in the horizontal plane, with two non-zero velocity components - radial $u_{r}$ and tangential $u_{t}$;

- the radial velocity component can be expressed in a classical way, namely by its mean value (averaged with respect to the reservoir depth $H$ ), varying from the outer wall to the central outflow:

$$
u_{t}(r)=\frac{Q}{(2 \pi r H)},
$$

- the tangential velocity can be calculated from the algebraic expression resulting from observations and measurements of this kind of flow (Rhodes 2008):

$$
u_{t}(r)=\frac{B}{r^{0.65}}
$$


- the semi-empirical multiplier $B$ can be calculated from the physical condition of equality of the inlet stream power and the power of energy dissipation during rotational flow, which yields:

$$
B=\frac{4.63 \cdot Q}{\left[H d_{i n}^{4} \cdot\left(\frac{1}{r_{w}^{0.95}}-\frac{1.41}{R^{0.95}}\right)\right]^{1 / 3}} .
$$

Averaged with respect to the radius $R$, tangential velocity equals:

$$
u_{t m}=2.86 B R^{-0.65} .
$$

Calculating the average velocity $u_{t m}$ for the known geometry of each pool and for the present discharge of water, one obtains (see Eqs. 11):

$$
u_{t m 3}=0.23 \mathrm{~m} / \mathrm{s} \quad u_{t m 4}=0.13 \mathrm{~m} / \mathrm{s} \quad u_{t m 7}=0.13 \mathrm{~m} / \mathrm{s} .
$$

These results are approximate but realistic, and therefore should be taken into account in these considerations, at least as supplementary information. The calculated values of the average velocity should be positively estimated, as they are high enough to improve mixing conditions in the system. The more so as the suspension contained in the breeding pools consists of very small particles, with a low sedimentation velocity. Moreover, the flow can be easily intensified by increasing the water discharge $Q$.

\section{Conclusions}

Water animals, including sea mammals, are usually kept in closed facilities. Still, people responsible for these animals ought to take care of their well-being by making living conditions in these facilities as close to the natural habitat as possible. If the animals remain in a completely artificial environment, this requirement is very difficult to fulfill.

A particularly important factor is the proper quality of water, which has to be ensured by water exchange. This can be done in different ways by different hydraulic systems. An especially important role is played by continuous water exchange in pools, which not only improves water quality, but also makes it possible to create proper dynamics of the velocity field. This dynamic factor is the subject of the paper, which is devoted to the analysis of the intensity of flow through the breeding pools in the sealarium in Hel.

The existing system in this sealarium has a standard hydraulic form. The inlets and outlets of water are perpendicular to the outer walls. In consequence, the velocity field is very poorly developed, with extended dead zones. The actual detention time in the breeding pools was determined experimentally by the tracer method. The results of measurements confirmed the theoretical expectation - the average detention time $t_{A}$ was incomparably shorter than the assumed plug-flow time $t_{P F}\left(t_{A}\right.$ is close to 0.03 $\left.t_{P F}\right)$. 
In order to increase the area of the active flow zone, an improvement of the hydraulic system was proposed. It was suggested that the inlets should be directed along the outer walls (i.e. the stream introduced into each pool should be parallel to the walls), whereas the outlets should be situated at the center of each reservoir (Fig. 4). Some calculations, based on a simplified model of rotational fluid motion, showed that even for the present water discharge the effective velocity in breeding pools should reach $0.1-0.2 \mathrm{~m} / \mathrm{s}$. This value should be sufficient to keep polluting particles in suspension.

These conclusions are somewhat general. A more accurate and extensive investigation of the problem described here would provide more information. Further research depends on the financial resources of the sealarium owner.

\section{References}

Andoh R. Y. G., Saul A. J. (2003) The use of hydrodynamic vortex separators and screening systems to improve water quality, Water Sci. Technol., 47 (4), 175-183. Amsterdam.

Danckwerts P. V. (1953) Continuous Flow Systems. Distribution of Residence Times, Chem. Engrg. Sci., 2, 1-13.

Gronowska-Szneler M. A. (2014) Dimensioning of vortex separator, PhD Dissertation, Gdańsk University of Technology, Gdańsk, Poland.

Gronowska-Szneler M. A., Sawicki J. M. (2014) Simple design criteria and efficiency of hydrodynamic vortex separator, Water Sci. Technol., 70 (3), 457-463.

Nalluri C., Featherstone R. F. (2001) Civil Engineering Hydraulics, Blackwell Science, Oxford, UK.

Rhodes M. (2008) Introduction to Particle Technology, John Wiley \& Sons Ltd., New Zealand.

Sawicki J. M. (1989) Kinematic models of plane flows, Proc. $3^{\text {rd }}$ Int. Symp. "Research on Hydraulic Engineering”, 18-20.09.1989, Gdańsk, Poland, 323-334.

Sawicki J. M., Skuza M. K., Bering S. (2003) Tracer investigations of dynamic properties of fluid-flow reactors, Proc. VIII Int. Symp. "Water Management and Hydraulic Engineering”, Podbanske 24-27.09.03, Slovak University of Technology, Bratislava, Slovakia.

Sawicki J. M. (2014) Computational fluid dynamics - lustres and shadows, Future Trends in Civil Engineering, University of Zagreb, Zagreb, Croatia, 62-77.

Wilson J. F., Cobb E. D., Kilpatrick F. A. (1986) Fluorometric Procedures for Dye Tracing, Techniques of Water-Resources Investigations of the US Geological Survey, 1-20. 